Gut, 1976, 17, 267-272

\title{
Endoscopic pancreatography in evaluating results of pancreatico-jejunostomy
}

\author{
CH. KUGELBERG ${ }^{1}$, L. WEHLIN, B. ARNESJÖ, AND U. TYLÉN \\ From the Departments of Surgery and Diagnostic Radiology, University Hospitals, Lund and Malmö, \\ Sweden
}

SUMMARY It has been difficult, employing clinical methods, to establish whether pancreaticoenteric drainage shunts for chronic pancreatitis have remained patent and how the operation has affected the inflammatory changes and ductal pathological morphology. In an attempt objectively to evaluate postoperative results, 13 patients with two different types of pancreatico-jejunostomies were examined with endoscopic pancreatography. Progress of the inflammatory changes was noted to a varying degree in the majority of cases. In four patients subjected to longitudinal split of the pancreatic duct, shunt patency was evidenced. In the remaining nine patients with caudal pancreaticojejunostomy, no contrast medium could be made to pass the shunts in six cases, whereas passage was clearly demonstrated in one and uncertain in two. All the patients reported clinical improvement after operation.

Hitherto, postoperative evaluation of different surgical drainage procedures for chronic pancreatitis has been largely limited to conventional clinical studies. The introduction of endoscopic retrograde cholangio-pancreaticography (ERCP) represents a new approach in this field, making it possible to compare the clinical results with ductal morphology and pathology.

This paper reports an attempt at objective evaluation of postoperative results utilizing ERCP in a series of patients operated upon for chronic and acute relapsing pancreatitis, with various types of pancreatico-jejunostomy.

The main interest will be focused on three issues:

1. Will pancreatico-jejunostomy preclude progress and even diminish the extent of the inflammatory process, as reflected by changes in the ductal system?

2. Will pancreatico-jejunostomies remain patent, providing the desired drainage facility ?

3. How will the results of postoperative pancreatography correspond with the patients' actual state of health?

\section{Methods}

During the decade 1963 to 1973,30 of the patients

'Present address: Lasarettet, Fach, S-261 20 Landskrona, Sweden. Received for publication 26 January 1976 treated for chronic or acute relapsing pancreatitis at Lund University Clinics had pancreatico-jejunostomies. Of these, 21 were operated upon with caudal shunts, end to side, after resection of the pancreatic tail according to DuVal (1954).

In the remaining nine, because of more advanced changes, the pancreatic duct was split longitudinally and anastomosed to a defunctionalized limb of the jejunum as elaborated by Puestow and Gillesby (1958).

The main indications for surgery were relief of pain and the hope of precluding progressive destruction of the pancreatic parenchyma and prevention of recurrent attacks.

After resection of various amounts of caudal pancreatic tissue, peroperative antegrade ductography was performed in all instances but one (Arnesjö et al., 1974).

A conventional clinical appraisal of long-term postoperative results in the entire series has recently been made (Arnesjö et al., 1975). In this survey, the patients were classified with regard to alcohol consumption, clinical evaluation of pancreatitis on operation, and severity of inflammatory reaction as found in peroperative pancreatography and postoperative microscopic analysis of resected specimens. The distribution of these findings in the group of patients relevant to the present study appears in Table 1.

Postoperatively, the patients were given a thorough 


\begin{tabular}{|c|c|c|c|c|c|c|c|}
\hline Patient no. & $\operatorname{Sex}$ & $\begin{array}{l}\text { Duration of } \\
\text { symtoms }(v r)\end{array}$ & $\begin{array}{l}\text { Alcohol } \\
\text { consumption }\end{array}$ & $\begin{array}{l}\text { Clinical } \\
\text { evaluation of } \\
\text { severity of } \\
\text { pancreatitis } \\
\text { at operation }\end{array}$ & $\begin{array}{l}\text { Severity of } \\
\text { pancreatitis as } \\
\text { found at } \\
\text { peroperative } \\
\text { pancreatography }\end{array}$ & $\begin{array}{l}\text { Severity of } \\
\text { pancreatitis as } \\
\text { found at } \\
\text { microscopic } \\
\text { analysis of } \\
\text { resected tail }\end{array}$ & Type of operation \\
\hline 1 & 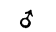 & 6 & +++ & ++ & ++ & $+t+$ & PG \\
\hline 2 & o & 4 & $+t+$ & $+t+$ & Not done & $++\div$ & PG \\
\hline 4 & 0 & 8 & $+t$ & $+1+$ & $++\ldots$ & ++1 & PG \\
\hline 5 & $\delta$ & 17 & $+t+$ & +++ & $++t$ & $++-t$ & $\mathrm{CP}$ \\
\hline 6 & s. & 2 & $+t+$ & + & + & $+\div$ & CP \\
\hline 7 & 8 & 10 & ++ & $+\div$ & ++ & + & $\mathrm{CP}$ \\
\hline 8 & 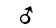 & 8 & ++ & $++\div$ & + & $+\div \div$ & $\mathrm{CP}$ \\
\hline 9 & 우 & 20 & + & $+t+$ & $+t$ & $+t$ & CP \\
\hline 10 & 0 & 18 & + & + & + & + & $\mathbf{C P}$ \\
\hline 11 & ㅇ & 10 & + & + & + & ++ & CP \\
\hline 12 & 5 & 7 & 0 & + & + & + & CP \\
\hline
\end{tabular}

Table 1 Degree of inflammatory pancreatic changes at clinical peroperative pancreatographic and microscopic examination, related to alcohol consumption and duration of symptoms

$+++=$ advanced $;++=$ moderate $+=$ mild $;-=$ normal; $\mathbf{C P}=$ caudal pancreatico-jejunostomy; PG $=$ Puestow-Gillesby procedure

clinical examination followed by an extensive interview. Routine analysis of blood and urine was performed as well. The summing up in each case was defined as excellent, good, fair, and poor, representing a summary of several criteria such as sense of wellbeing, disappearance or relief of pain, improvement of nutritional state, resumption of work, need for continuous medication or rehospitalization. Postoperative evaluation of exocrine function was omitted since collection of pancreatic secretion is technically difficult from the shunted gland. A trial was made with serial scintigraphs before and after hormone stimulation, but the results were difficult to interpret.

An additional survey of the entire series has been made by an independent group from the Institution of Sociology at the University of Lund. The results of the two studies correspond very well and form the basis of clinical evaluation in the present study which was performed approximately one year later. No changes in subjective postoperative results were noted.

Twenty-two of the patients accepted a postoperative ERCP on a voluntary basis. In 14 of those, cannulation attempts were successful. All cannulations were done at Malmö General Hospital using the Olympus JF-B or JF-B2 duodenoscope. As premedication Valium 5-10 mg i.v. were employed. Pharynx anaesthesia was achieved with $4 \%$ Xylocain spray, and bowel peristalsis was controlled with Buscopan administered i.m. and/or i.v. The contrast medium used was Urografin $60 \%$ in 10 cases and Isopaque 350 in the remaining four.

After cannulation and injection of contrast medium the patient was rotated from half prone position, right side up, to the supine, whereupon films were exposed in various projections.

The examination was terminated taking delayed pictures, checking the emptying process. Shunt patency, as evidenced by passage of injected contrast medium, was judged by at least two persons closely observing the procedure using fluoroscopy. In six cases the entire procedure was recorded on video tape.

The contrast medium entered the pancreatic duct alone in six cases, whereas the biliary as well as the pancreatic duct system were evidenced in the remaining eight. In one of these latter patients an anatomical variant of the pancreatic duct system was observed as described by Millbourn (1950) and classified as type ' $\mathrm{S} 2$ '.

In this variant the duct of Santorini is the main excretory channel while the duct of Wirsung drains only a restricted section of the parenchyma without any connection with the principal duct system. On cannulation of this patient, the catheter entered the small duct of Wirsung which was filled with contrast medium together with the biliary duct system. The duct of Santorini was not cannulated and therefore information concerning the state of the main excretory duct in this patient was not obtained.

Apart from elevations of serum amylase, without clinical symptoms, in four patients, no complications were noted except in one case. This was the abovementioned patient with the anatomical variant classified as ' $\mathrm{S} 2$ '. On injection, contrast medium was extravasated into a restricted area of pancreatic parenchyma. Later the patient developed jaundice, diabetes mellitus and bilateral peroneus paresis. The cause of this development remains obscure. No 
obstruction to bile flow could be shown by PTC or on operation performed because of increasing jaundice. The patient recovered after steroid treatment, indicating that some immunological reaction was responsible for the symptoms.

This case is excluded from our final study, which thus consists of 13 patients. Four of these were operated upon according to Puestow and Gillesby (1958) with longitudinal split while nine had caudal pancreatico-jejunostomies (Table 1).

\section{Results}

In the group of four patients operated upon with longitudinal split of the pancreatic duct, two cases exhibited progress of the ductal inflammatory changes, such as further dilatation, irregularity and formation of intraluminal concretions (Fig. 1). Concerning the remaining two patients, the short observation time did not permit any conclusions in one case and no peroperative pancreatography had been performed in the other. Shunt patency was evident in all four cases on postoperative ductography (Fig. 1). The mean observation time between per- and postoperative ductography was one year and two months.

In the nine patients where caudal pancreaticojejunostomy had been performed, definite signs of progress of inflammatory ductal changes were noted in six cases. No obvious progress was seen in two, whereas the findings were difficult to evaluate in one. In some cases the signs of progress were quite pronounced (Fig. 2). Shunt patency, as evidenced by the passage of contrast medium, was obvious in one case

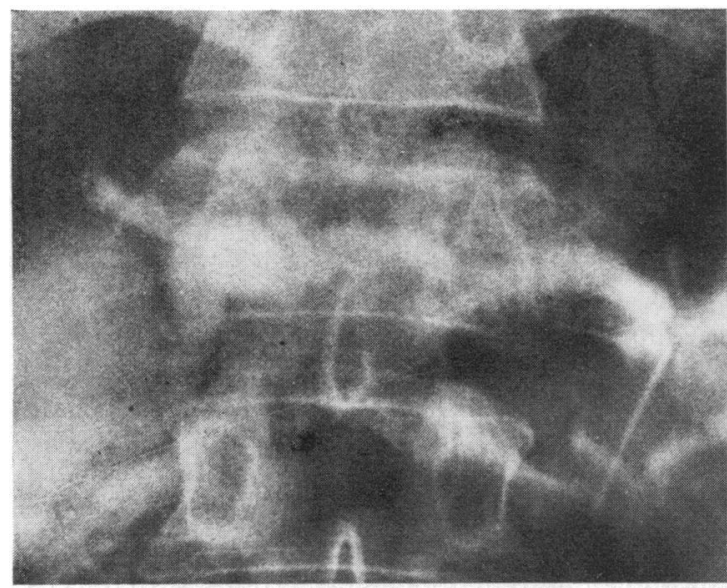

(a)

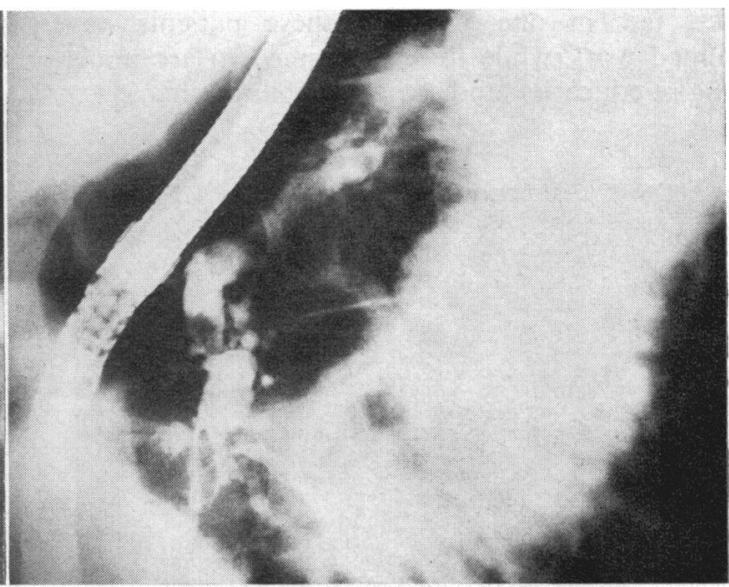

(b)

Fig. 1 Puestow-Gillesby shunt. (a) Peroperative pancreatography. Advanced pancreatitis. (b) Two years postoperatively. Progress with formation of concretions and strictures. Shunt patent.

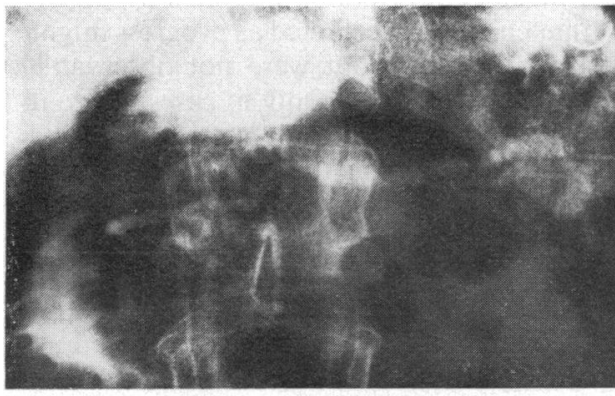

(a)

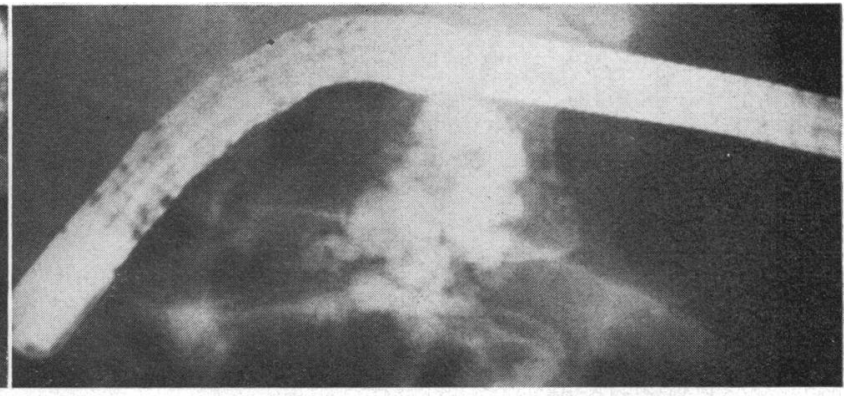

(b)

Fig. 2 Caudal pancreatico-jejunostomy. (a) Peroperative pancreatography. Rather modest ductal changes. (b) Pronounced progress after one year with ductal dilatation and concretions in the pancreatic head. Occlusion of the main duct at the borderline head/body making evaluation of shunt patency impossible. 
(Fig. 3), and uncertain in one. In an additional patent shunt, patency could not be evaluated as a stricture had developed in the corpus constituting an obstacle for further passage of the contrast medium (Fig. 2). In the remaining six patients no contrast medium could be made to pass the shunts, suggesting occlusion (Fig. 4). The mean observation time between ductographies was three years and one month.

Intraluminal concretions had formed postoperatively in six cases, five of which were operated upon with caudal shunts and one with longitudinal split (Fig. 5).

From a clinical point of view postoperative results were encouraging. Among the patients with longitudinal splits, three admitted undue alcohol consumption postoperatively, despite full information of the risks. Still, they claimed definite clinical improvement as a result of the operation-excellent in three cases, fair in one. Two of these patients have resumed work while the remaining two are undergoing re-education and social rehabilitation.

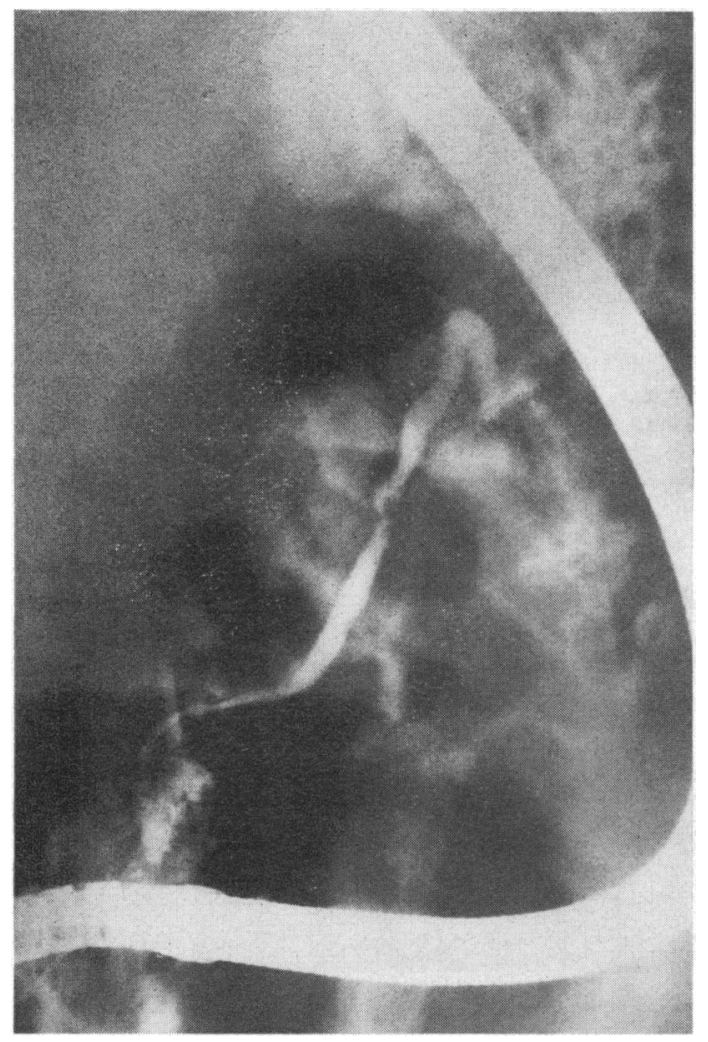

Fig. 3 Caudal pancreatico-jejunostomy. Postoperative pancreatography. Advanced pancreatitis. Shunt patent.
Of the nine patients with caudal pancreaticojejunostomy, one has ceased drinking alcohol altogether, one has reduced consumption considerably, two have increased their intake, while the remaining five state unaltered drinking habits. Six of these patients claimed excellent results of the operation, two claimed good results, and one fair. Five of these nine patients have resumed their previous occupations.

Related parameters of interest appear in Table 2.

\section{Discussion}

It is interesting to note the high incidence of unsuccessful cannulation attempts in the patients of our series as compared with patients not subjected to surgery in the actual field in whom our success rate was $90 \%$ in 189 examinations during the same period. Technical difficulties caused by the previous operative procedures seem to be the main reason for the failures.

Most of the postoperative ductographies indicated obvious progress of the inflammatory changes. In evaluating these findings it must be borne in mind that the peroperative antegrade and the postoperative retrograde injection of contrast medium represent two technically different methods of examination.

This fact may sometimes cause difficulties in deciding whether progress has occurred or not. ERCP will yield more detailed information concerning ductal morphology than the antegrade examination performed during operation. This may well be explained by the fact that in ERCP contrast medium is injected under fluoroscopic control permitting optimal filling of the duct system and the exposure of films in optimal projections, with full cooperation from the patient. When performing the examination during operation, the patient is under general anaesthesia, fixed to the operating table. Facilities of fluoroscopy are not available.

The more detailed information obtained by ERCP may imply that changes interpreted as progress might have existed at operation but were not observable with the technique employed. Only in cases where it has been possible to exclude these sources of error with reasonable certainty has the occurrence of progress been assumed.

At times it was difficult to determine whether the shunt was patent. If deep, selective catheterization of the main pancreatic duct was achieved, no problems were encountered. In other cases the procedure was disturbed by reflux of contrast medium to the duodenum or by remaining contrast medium in the bowel after unsuccessful cannulation attempts. The best observations were generally made during the first injection of contrast medium with the patient 

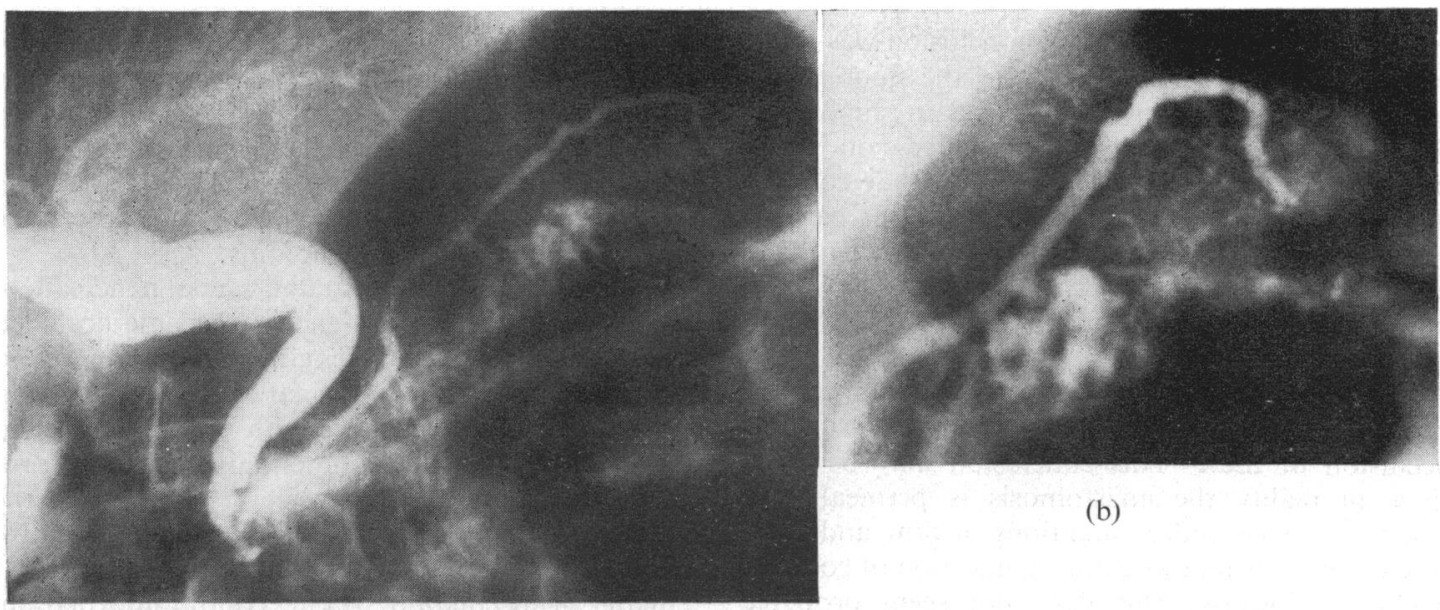

(b)

(a)

Fig. 4 Caudal pancreatico-jejunostomy. Postoperative pancreatography after 3.5 years. Mild pancreatitis. Shunt occluded. (a) Both duct systems contrast filled. Bile tree dilated (cholecystectomy). (b) Spot film of tail showing occlusion. Note filling of small tributaries and increasing density of parenchyma.

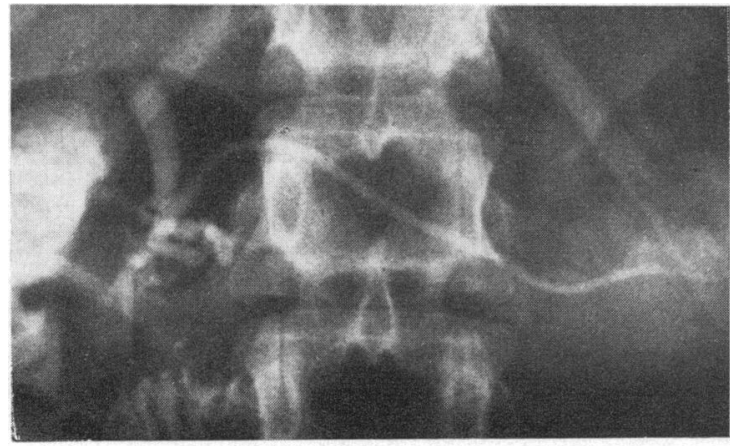

(a)

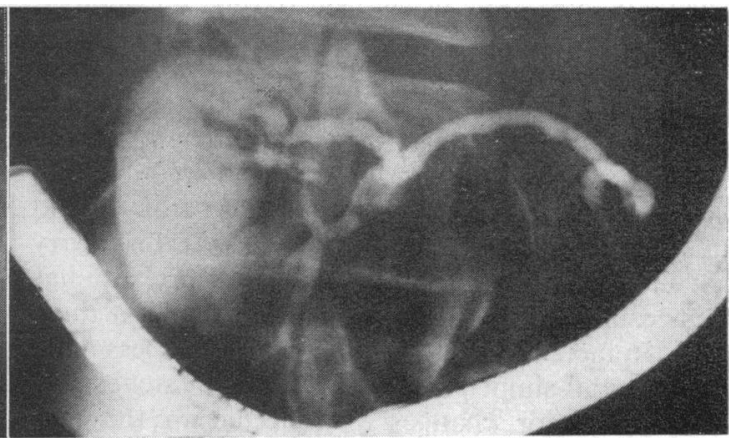

(b)

Fig. 5 Caudal pancreatico-jejunostomy. (a) Peroperative pancreatography. Mild pancreatitis. (b) Progress after three years with formation of a large concretion in the main duct.

\begin{tabular}{|c|c|c|c|c|c|c|c|}
\hline Patient no. & $\begin{array}{l}\text { Type of } \\
\text { operation }\end{array}$ & $\begin{array}{l}\text { Overall subjective } \\
\text { long-term post- } \\
\text { operative results }\end{array}$ & $\begin{array}{l}\text { Time of } \\
\text { postoperative } \\
\text { follow up } \\
\text { (months) }\end{array}$ & $\begin{array}{l}\text { Span of time } \\
\text { between per-and } \\
\text { postoperative } \\
\text { pancreatography } \\
\text { (months) }\end{array}$ & $\begin{array}{l}\text { Pre-/post- } \\
\text { operative alcohol } \\
\text { consumption }\end{array}$ & $\begin{array}{l}\text { Intraductal } \\
\text { concretions } \\
\text { pre-/postop. }\end{array}$ & $\begin{array}{l}\text { Postoperative } \\
\text { shunt patency }\end{array}$ \\
\hline 1 & PG & Fair & 30 & 20 & $+t+1++t$ & $0 /+$ & + \\
\hline 2 & PG & & 18 & 7 & $+++1++$ & $+1+$ & + \\
\hline 3 & PG & Excellent & 42 & 25 & $+t+1+t+$ & $+1+$ & + \\
\hline 5 & $\mathrm{CP}$ & & 42 & 29 & $+t+1+$ & $0 /+$ & + \\
\hline 6 & CP & Good & 42 & 31 & $+++1+++$ & $0 / 0$ & $?$ \\
\hline 7 & CP & & 114 & 106 & ++10 & $\mathbf{0} /+$ & - \\
\hline 8 & $\mathbf{C P}$ & Excellent & 18 & 13 & $++1++t$ & $\mathbf{0} /+$ & $?$ \\
\hline 9 & CP & & 24 & 14 & $+1+$ & $+1+$ & - \\
\hline 10 & CP & & 54 & 43 & $+1+$ & $0 / 0$ & - \\
\hline 11 & $\mathrm{CP}$ & Fair & 30 & 18 & $+1+-$ & $0 /+$ & - \\
\hline 12 & $\mathrm{CP}$ & Good & 42 & 42 & $0 / 0$ & $0 / 0$ & - \\
\hline 13 & $\mathrm{CP}$ & Excellent & 66 & 38 & $0 / 0$ & $0 /+$ & - \\
\hline
\end{tabular}

Table 2 Subjective postoperative results related to shunt patency and intraductal concretions

Concerning intraductal concretions a single pius signifies existence. In case no. 2 existence of stones was estimated at surgery, as no peroperative pancreatography was performed. 
lying on his left side. The final conclusion was based on the impressions obtained from the fluoroscopic observations, the exposed films and in some cases the video-tape recordings. Founded on these grounds, the conclusion was reached that contrast medium passed the anastomosis in all four patients with longitudinal splits whereas it appeared to pass only one of the caudal shunts, passage being uncertain in additionally two and, in all probability, nonexistent in the remainder.

The apparent non-passage of contrast medium over practically all the caudal shunts suggests occlusion of these. This conclusion may be false, since in reality the anastomosis is permeable to pancreatic juice under conditions of flow and pressure different from those during injection of contrast medium. However, this does not seem probable considering the frequency of the phenomenon and the good quality of the pancreatograms, visualizing even minute tributaries (Fig. 4b). The slight difference of viscosity between the contrast medium and pancreatic juice does not appear to offer an explanation.

Accepting the fact that the shunts have occluded, interest focuses on the reasons for the occlusion. Most workers agree that the symptoms and pathophysiological changes in chronic pancreatitis owe their origin to obstruction of pancreatic outflow and subsequent intraductal pressure peaks. If this theory be valid, it may be that the intraductal pressure has been insufficient to keep the shunts patent in these cases. In fact, all patients subjected to the less traumatic caudal shunt procedure had slight stenosis or relatively minor changes as reflected in the peroperative pancreatograms. Then the question arises whether these patients were wrongly selected for caudal pancreatico-jejunostomy.

At the present stage no definite answer can be given to this question as our examinations reflect only the conditions at a given moment. It is unknown for how long the shunts remained patent postoperatively and the actual benefit hereof. Follow-up with repeated postoperative ERCP examinations at controlled intervals will prove informative in this matter.

Diametrically opposed to the above mentioned disappointing results is our definite impression that the operation has had a most positive effect on the general sense of well-being in these patients. Objective signs of improvement have also been recorded. Before surgery, all patients had had at least six welldefined attacks of pancreatitis requiring hospital care. Postoperatively, only one has had recurrence of pancreatitis, occurring three months after the operation. On exploration, an obstruction, probably due to a small calculus, was removed. Progress of the inflammatory changes was noted as well. Reanastomosis was performed and thereafter the patient has been free of symptoms.

To sum up, it would seem that caudal pancreaticojejunostomies have a tendency to become occluded while anastomoses after longitudinal splits seem to remain open. Progress of ductal changes was seen in most patients, also in those with open shunts, suggesting that even a well-functioning drainage procedure does not stop or reverse the advance of the disease. Whether the operation has had a moderative influence on the velocity of inflammatory progress is a matter of speculation, lacking control information. The continuation of the progress appears to be independent of postoperative alcohol consumption. Nevertheless, the operations seem to have relieved our patients from recurring attacks of pancreatitis and, to a large extent, abolished upper abdominal pain. Further research is required to explain this contradiction.

Viewed from this aspect, pancreatico-jejunostomy for chronic or acute relapsing pancreatitis is not a curative measure, but it may be regarded as palliative surgical intervention. There also seems to be room for doubt as to whether the ductal changes really are secondary to increased pressure caused by obstruction of pancreatic outflow. It seems more reasonable that the ductal changes constitute a part of the inflammatory process engaging the entire exocrine pancreas, progressive when once initiated.

\section{References}

Arnesjö, B., Kugelberg, C., and Tylén, U. (1974). Pancreatography in relapsing pancreatitis. Acta Chirurgica Scandinavica, 140, 131-137.

Arnesjö, B., Ihse, I., Kugelberg, C. F., and Tylén, U. (1975). Pancreatico-jejunostomy in chronic pancreatitis. Acta Chirurgica Scandinavica, 141, 139-148.

DuVal, M. K. Jr. (1954). Caudal pancreatico-jejunostomy for chronic relapsing pancreatitis. Annals of Surgery, 140, 775-785.

Millbourn, E. (1950). On the excretory ducts of the pancreas in man, with special reference to their relations to each other, to the common bile duct and to the duodenum. A radiological and anatomical study. Acta Anatomica, 9, 134.

Puestow, C. B., and Gillesby, W. J. (1958). Retrograde surgical drainage of pancreas for chronic relapsing pancreatitis. Archives of Surgery, 76, 898-907. 